\section{Using Soft Computing Techniques for Improving Foot Trajectories in Walking Machines}

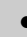 \\ -}

\author{
Elena Garcia*, Pablo Gonzalez-de-Santos \\ Instituto de Automatica Industrial-CSIC \\ La Poveda, 28500 Arganda del Rey \\ Madrid, Spain
}

Received 30 May 2000; accepted 27 February 2001

\begin{abstract}
Walking machines have been investigated during the last 40 years and some basic techniques of this field are already well known. However, some aspects still need to be optimized. For instance, speed seems to be one of the major shortcomings of legged robots; thus, improving leg speed has been chosen as the main aim of this work. Although some algorithms for optimizing trajectory control of robot manipulators already exist, we propose a more computationally efficient method that employs fuzzy set theory to involve real dynamic effects over leg motion instead of an inaccurate mathematical model. In this article, we improve leg speed by automatically tuning the acceleration of legs. For this purpose, we define fuzzy rules based on experiments and we find the optimal acceleration for every given trajectory. A simple fuzzy inference system is used to compute the required acceleration. It is based on five rules using three linguistic variables. Final results show that foot acceleration tuning for straight trajectory generation is a suitable method for achieving accurate, smooth and fast foot movements. Also it is shown that under some conditions average leg speed can be increased up to $100 \%$ using the control methods herein proposed. ๑ 2001 John Wiley \& Sons, Inc.
\end{abstract}

\section{INTRODUCTION}

Legged robots have been extensively investigated during the last four decades, and activity in this field is still increasing. Work in these years was mainly

* To whom all correspondence should be addressed; e-mail: egarcia@iai.csic.es.

Contract Grant Sponsor: CICYT (Spain).

Contract Grant number: TAP1999-1080-C04-01. concentrated on the design of leg mechanisms and machine structures, and some areas of knowledge are quite well known now, such as the generation of continuous, ${ }^{1}$ discontinuous ${ }^{2}$ and free $^{3}$ statically stable gaits. There is also great activity in the understanding of living walking locomotion. Zoologists and biologists are becoming interested in this technology and they are trying to apply biological understanding to the design and control of walking machines. ${ }^{4,5}$ 
Currently there are about 100 walking machines all over the world. Most of them are simple laboratory prototypes; however, there are real needs for walking machines. Although industry is timidly incorporating walking machines in industrial processes, 6,7 there are still many potential non-industrial applications for walking machines, such as terrestrial and planetary exploration ${ }^{8}$ and humanitarian de-mining. Nevertheless, in order to accomplish successful real applications, some problems must be solved, and the current performance of walking machines must be improved.

Detractors of legged robots usually point out machine speed as one of the major shortcomings of these vehicles. Machine speed, mainly depends on foot speed and assuming that a leg is indeed a 3 DOF manipulator, there is no apparent reason for building legs with features far differing from manipulator features. Modern industrial manipulators exhibit great features such as speed, repeatability and accuracy for accomplishing industrial applications, but a walking machine leg differs from a manipulator in too many important characteristics. First, legs cannot sweep big volumes during the leg-transfer phase or while propelling the body, so they must be slender devices. Furthermore, legs must carry bigger payloads than manipulators. For instance, a given commercial manipulator designed to carry a 3-kg payload weighs about $50 \mathrm{~kg}$ and exhibits a strong structure. However, a leg for the SILO4 walking robot, used for experiments in this article, weights $4 \mathrm{~kg}$ and must support, for some foot positions, up to $15 \mathrm{~kg}$ (half the machine's weight). A similar comparison can be done with well-known machines such as the $\mathrm{ASV}^{1}$ or $\mathrm{AMBLER}^{8}$ As a consequence, legs may be said to be weaker than manipulators.

Manipulators are extremely accurate devices that need to be rigid, and as a result they are bulky. A leg does not need great accuracy in placing its foot at a given position. Normally, positioning errors of a few centimeters are allowed in walking with static stability. Nevertheless, when the body is propelled in any direction, we get up to $n$ cooperating legs that must move their contact point with the body along perfect straight and parallel lines ( $n$ is the number of legs of the machine). Otherwise, some internal forces will appear and cause foot slippage or joint flexure and link bending. In any of these cases the body will exhibit undesired motion.

Summarizing, a walking machine's leg must be designed to work under worse-load condition than a manipulator, with a weaker mechanical structure and achieving good accuracy. Normally, the big payloads of legs are carried using strong gearing; thus legs become slower than manipulators. Therefore, leg velocity is a feature that must be carefully optimized.

This article is focused on the optimization of leg velocity for real-time trajectory tracking. Optimization of speed in robot manipulators has been extensively studied in the last two decades. Bobrow et al. ${ }^{9}$ and Shin and McKay ${ }^{10}$ independently developed similar methods to find the minimumtime control of serial manipulators along specified paths with actuator torque limitations. To compute the mentioned optimal control problem, dynamic equations of the manipulator were reduced to a set of second-order differential equations in a path parameter, and the bounds on actuator torques were transformed to the bounds on the acceleration along the path. Finding the numerical solution of such time-optimal control problems is computationally very intensive. Their proposed method was revised and more efficient algorithms were presented in later publications. ${ }^{11-13}$ Slotine and Yang ${ }^{12}$ found computational improvements over the existing methods, making quasi-real-time trajectory generation feasible for a hypothetical planar two-link manipulator. There is no evidence that the proposed algorithms could be applicable for real-time trajectory generation of a higher number of degrees of freedom manipulator.

Every existing algorithm finding time-optimal solutions to trajectory tracking in robot manipulators points out the relevant effect of robot dynamics over the motion along the path. Moreover, the minimum-time control theory assumes that a perfectly accurate manipulator model is available and that there are no external disturbances. In practice, however, it is not possible to obtain such an ideal model. Furthermore, assuming a non-ideal mathematical model of the manipulator is found, several assumptions have to be made in order to simplify the model if real-time programming is required (i.e., absence of friction and other disturbances, diagonal mass matrix, etc.). As a result, an inaccurate model of the system will be employed and errors will be found during real application. Yang and Slotine ${ }^{14}$ developed near-minimum-time control algorithms that coped with the applicability limitations of classical methods. Their proposed method found a compromise between computation time and speed optimization. It also required a model of the robot, but managed better with mathematical inaccuracies 
due to its closed loop structure. The method generated near-minimum-time solutions within $0.5 \mathrm{~s}$ on a DEC 5000/25 workstation for a two-link manipulator; however, the order of computational burden increases as the number of degrees of freedom increases.

In this article the authors propose a method to find optimal speed of trajectory tracking for the leg of a walking machine. The SILO4 walking robot, described in section 2 , is used as a testbed for testing the developed algorithms. The method proposed is computationally efficient and its real-time implementation for on-line path generation is proved. The proposed algorithm does not include a mathematical model of the robot leg to avoid errors due to mathematical simplification. It includes the real dynamic effects existing during the leg motion instead. The dynamics affecting the leg motion were carefully analyzed in previous work ${ }^{15}$ and results are introduced in this algorithm as fuzzy rules reflecting the existing dynamics. Since rules are imposed on the foot motion, the computational complexity of the algorithm is independent on the number of degrees of freedom of the robot leg.

The application of fuzzy set theory is especially recommended when a mathematical model of the system is not available. In the authors' opinion, it provides an efficient representation of the real dynamic effects over the system motion. Fuzzy rules also provide a good representation of parameter uncertainties existing on real machines. Fuzzy logic techniques have been recently applied to manipulators ${ }^{16}$ and individual axis control, ${ }^{17}$ and they have proved to be an adequate tool for designing control methods.

This article addresses the optimization of leg velocity in walking machines using fuzzy techniques. The phenomenon that motivated this work is explained in the results obtained experimentally with a small walking robot, which is used as a testbed for method evaluation as well. Nevertheless, the algorithms presented in this article are general methods applicable to any machine.

For a better understanding of this work, section 2 briefly introduces the SILO4 walking robot. Then, section 3 states the problem using some real examples. This problem is solved by fuzzy rules in section 4. Finally, experimental results are reported in section 5 .

\section{EXPERIMENTAL TESTBED}

The walking machine used in the modeling and experiments in this article was designed as a small robot for education and basic research purposes. Although there are some legged robots in the market for research and education, they are basically toys with very low ground-adaptation capability and very weak mechanical construction. The main idea behind this new development is to configure a small, easy-to-handle, reliable walking robot with great terrain-adaptability features, to be used as a comparative testbed of new methods and algorithms. Taking into account these considerations, the IAI-CSIC, based on its experience in the construction of several walking machines (RIMHO, ${ }^{2}$ ROWER, $\left.{ }^{6} \mathrm{REST}^{7}\right)$, decided to build the new robot SILO4 (Spanish acronym for Locomotion System). This robot is expected to become a common testbed for experiments and discussion on subjects such as artificial intelligence, perception integration, motion generation, etc. Currently there are two SILO4 robots being used as a comparative testbed in two different working groups (IAI-CSIC and University of Bourges (ENSI), France).

The leg of the SILO4, shown in Figure 1, is based on an insect-like leg formed by slender links that contain the actuators. Each link is about $0.238 \mathrm{~m}$

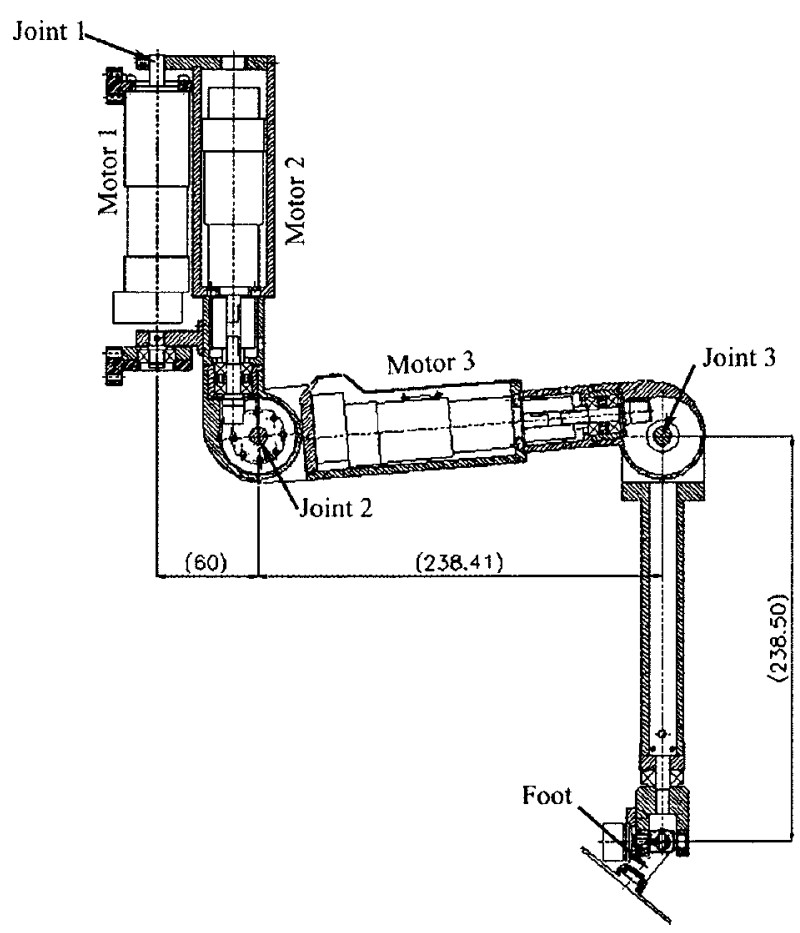

Figure 1. General view of the SILO4 leg configuration. 


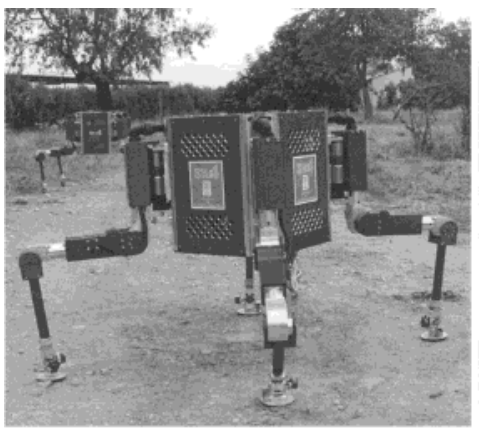

(a)

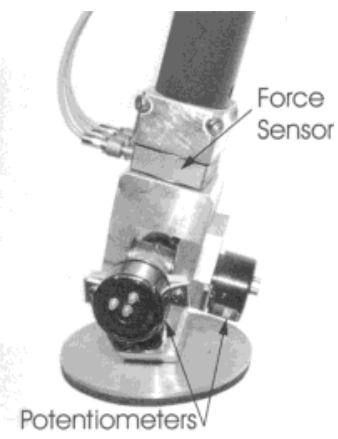

(b)
Figure 2. The SILO4 walking robot and (b) foot detail.

long, and the leg features an average speed of about $0.4 \mathrm{~m} / \mathrm{s}(0.2 \mathrm{~m} / \mathrm{s}$ when the foot follows a straight line). The leg is based on three rotary joints actuated by three DC motors with planetary and skew-axis gears. This leg has a special articulated three-passive-joint foot consisting of a universal joint (two passive joints) with a force sensor and a couple of potentiometers to measure joint orientation when the sole (another passive joint) is in its support phase (see Fig. 2b). The position sensors help in finding the components of the force acting on the foot. ${ }^{18}$

The SILO4 walking robot was designed to have the following features:

- four legs;

- small size and low weight, for ease in handling;

- mechanically robust;

- slender: avoiding motor positions that result in big leg volumes;

- compact: all motors and electrical cables conveniently housed;

- agile in changing trajectories; good omnidirectionality;

- controlled by a true real-time multitasking operating system supporting network communications.

The body is an irregular octagonal prism that contains the main computer, joint controllers and joint drivers. The four legs are attached to the four small walls in a circular configuration (see Fig. 2a). Table I summarizes some of the robot's features.

As a basic research prototype, the SILO4 robot is a mechanical system open to any controller. The controller used in the experimental work presented in this article consists of a PC-based host computer with four 3-axis control boards that perform position
Table I. Features of the SILO4 walking robot.

\begin{tabular}{llcc}
\hline & Dimension $(\mathrm{mm})$ & Speed $(\mathrm{mm} / \mathrm{s})$ & Mass $(\mathrm{kg})$ \\
\hline Body & Height 300 & 200 & 18 \\
& Width 310 & & \\
& Length 310 & & \\
Leg & 238 (each link) & 200 & 4 \\
Machine & & & 34 \\
Payload & & $\approx 10$ \\
\hline
\end{tabular}

control for all 12 of the robot's joints. The 12-DC motor drivers are based on the PWM technique. The host computer runs the QNX multitasking and realtime operating system.

\section{PROBLEM APPROACH}

One important feature dealing with kinematic chains of articulated joints appears when attempting to describe a straight-line trajectory of the end-effector. Every measured magnitude of a particular trajectory (i.e., traveled distance, average speed, end-effector acceleration) belongs to the Cartesian coordinate frame, while it must be mapped into the articulated joint space to accomplish the movement. This means that angular joint speeds must be synchronized to obtain a given linear end-effector speed. However, the joint speed value required for a desired endeffector task might be unreachable due to limitations of the joint drive. ${ }^{19}$ This problem arises especially in legged locomotion. Legs must be light and agile and must produce accurate movements. However, some leg configurations need to confine joint actuators. Thus, joint drives in such walking machines cannot be heavy, and this stricture directly affects their power. The problem of improving end-effector trajectories, and the results presented in this article for trajectory speed optimization, can be applied to any articulated manipulator; this article, however, will focus on legged locomotion, where speed optimization is a clear need.

In order to focus better on the problem of improving foot trajectories, let us define a plane formed by the straight trajectory of the foot and the origin of the leg reference frame. Let us label it plane IOF (see Fig. 3). The straight movement of the foot inside the plane IOF is characterized by the following parameters: The initial position, (I); the final position, $(\mathrm{F})$; the trajectory distance, $s(t)$; the foot speed, $\nu_{\text {foot }}(t)$; the angular displacement, $\theta(t)$, and its first derivate, $\dot{\theta}(t)$, which is the foot angular speed; 


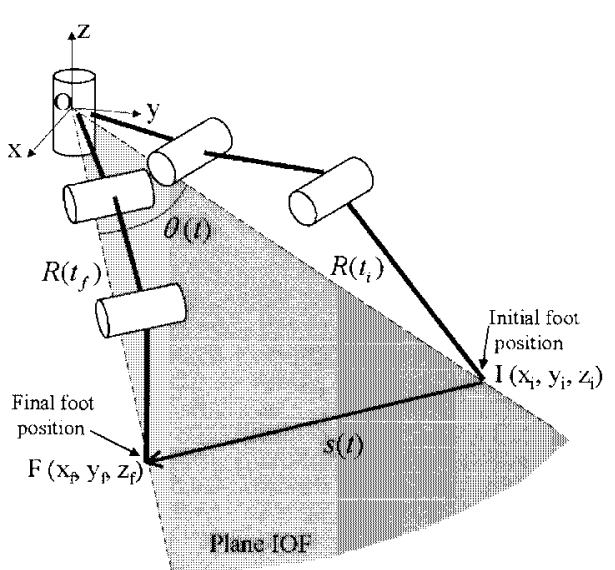

Figure 3. Foot trajectory and plane of motion.

and a variable radius, $R(t)$, which depends on the leg's kinematic configuration (see Fig. 3). Evident relationships exist among these parameters, but the relationship of interest here is the one that restricts the linear foot speed, $\nu_{\text {foot }}$. Obviously, the foot angular speed, $\dot{\theta}(t)$, and the radius, $R(t)$, will limit the maximum foot speed value for a given straight-line trajectory, that is,

$$
\nu_{\text {foot }} \leq \dot{\theta}_{\max } \cdot \min _{t_{i} \leq t \leq t_{f}}\{R(t)\}
$$

Let us assume that the foot angular speed, $\dot{\theta}(t)$, in the plane IOF is limited by the maximum driving speed of one of the three joints of the leg. Then, according to Eq. (1), the linear foot speed will be limited by the angular speed of this joint for trajectories where $R(t)$ is small at any instant of time, $t$. Thus, the linear foot speed is drive-limited at a given instant of time during trajectory execution, and consequently average foot speed, $\left(\nu_{m}\right)$, will also be restricted. However, average foot speed would not be drive-limited for trajectories with higher values of $R(t)$. Increasing average trajectory speed is the main goal of the work presented in this article.

After this observation, let us consider two types of trajectories inside the leg workspace. The first type will be trajectories of small $R(t)$, whose maximum average foot speed will depend largely on motor speed. There is no other possibility for increasing average foot speed for such trajectories than incorporating more powerful drives, despite the increase in leg weight if the leg encloses the joint drives. The second type of trajectory will be trajectories of high $R(t)$, which could reach very high foot speeds. If the straight line is generated using a trapezoidal velocity profile with a given

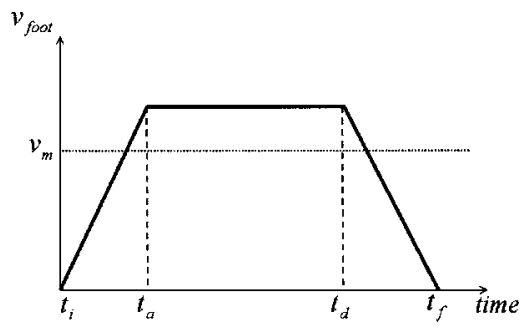

Figure 4. Trapezoidal foot-velocity profile for straightline generation. $\nu_{m}$ is the average foot velocity and $t_{\mathrm{a}}$ and $t_{\mathrm{d}}$ are acceleration and deceleration times, respectively.

foot acceleration ${ }^{20}$ (see Fig. 4), then the only magnitude that can limit foot speed for the second type of trajectory is the acceleration of the velocity profile. Hence, increasing the average foot speed in that type of trajectory can be achieved by increasing foot acceleration. However, increasing the acceleration of the velocity profile is not always an admissible solution. Leg dynamics could prevent the desired trajectory for a step-velocity profile with infinite acceleration from being followed. In this article we will try to find the appropriate velocity profile for each particular type of trajectory, taking into account the effects of leg dynamics without using complex techniques of dynamic control. It is worthwhile to use this procedure to improve leg performance in view of some of the current walking machine drawbacks pointed out in section 1 .

To illustrate this explanation, Figures 5 and 7 show how angular joint speeds affect the average foot speed in an articulated leg such as the SILO4 leg example. Figure 5(a) shows angular speed profiles of the first joint of the SILO4 leg for trajectories that are parallel to the leg's $x-y$ plane, for different values of $R(t)$. In this particular case the value $\dot{\theta}_{\max }$ of Eq. (1) corresponds to the first joint speed, the plane IOF is parallel to the leg's $x-y$ plane, and the value of $R(t)$ increases with $y$ component in the leg's Cartesian reference frame. Figure 5(a) shows that joint speed reaches its maximum driving speed for short values of $R(t)$, while it decreases for trajectories with higher values of $R(t)$. As stated in Eq. (1) (see Fig. 5b), foot speed evolution for the same trajectories is limited by the first joint speed for small values of $R(t)$. On the other hand, foot speed for higher values of $R(t)$ could be increased until it reaches a triangular footvelocity profile or could even better this value by increasing foot acceleration (see Fig. 6). Speed profiles of the second and third joints of the leg do not affect foot-velocity profiles in this example and are not depicted in Figure 5. 


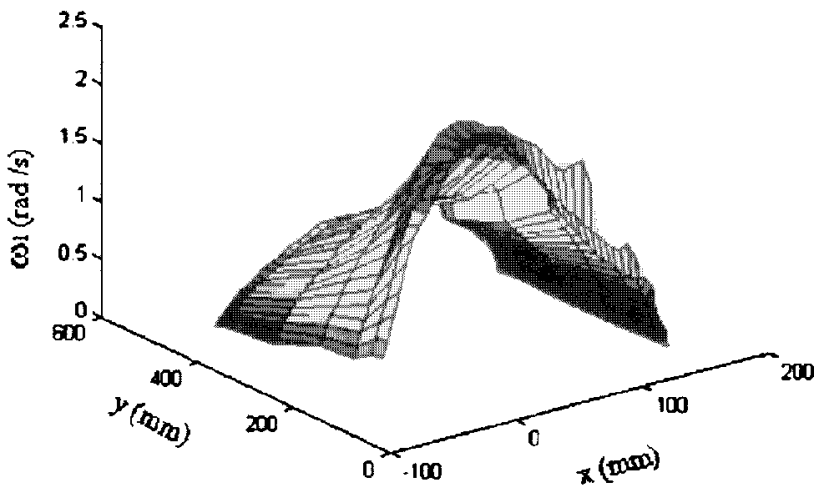

(a)

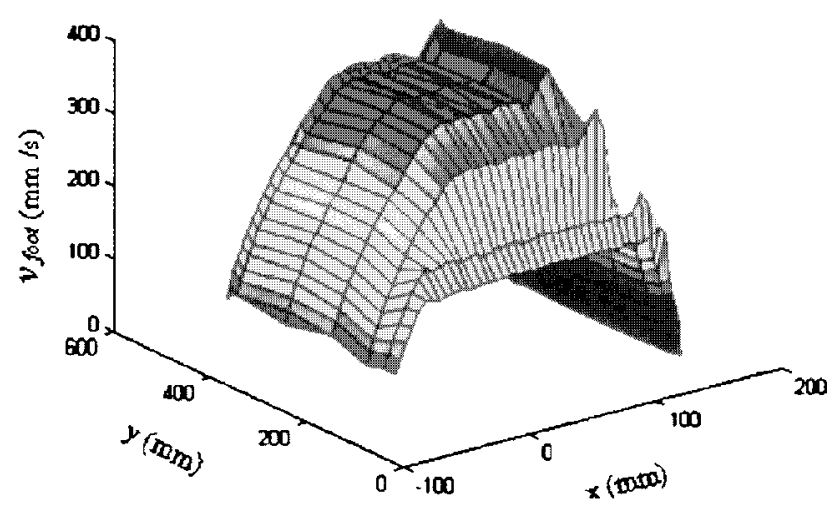

(b)

Figure 5. Foot trajectories parallel to the leg $x-y$ plane. (a) First angular joint speed profile evolution. (b) Foot velocity profile evolution.

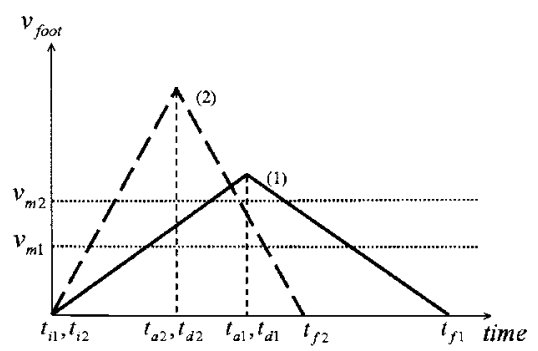

Figure 6. Two foot-velocity profiles for the same distance traveled during straight line generation.

Using foot-velocity profiles with higher acceleration might help achieve higher foot speed in another situation. Foot-velocity profile becomes triangular for short trajectories (small $s(t)$ ), and hence, only small average foot speeds can be achieved. Increasing foot acceleration would allow short trajectories to be performed at high speeds. Figure 6 shows two foot-velocity profiles for the same distance traveled. Profile (1) has lower foot acceleration than profile

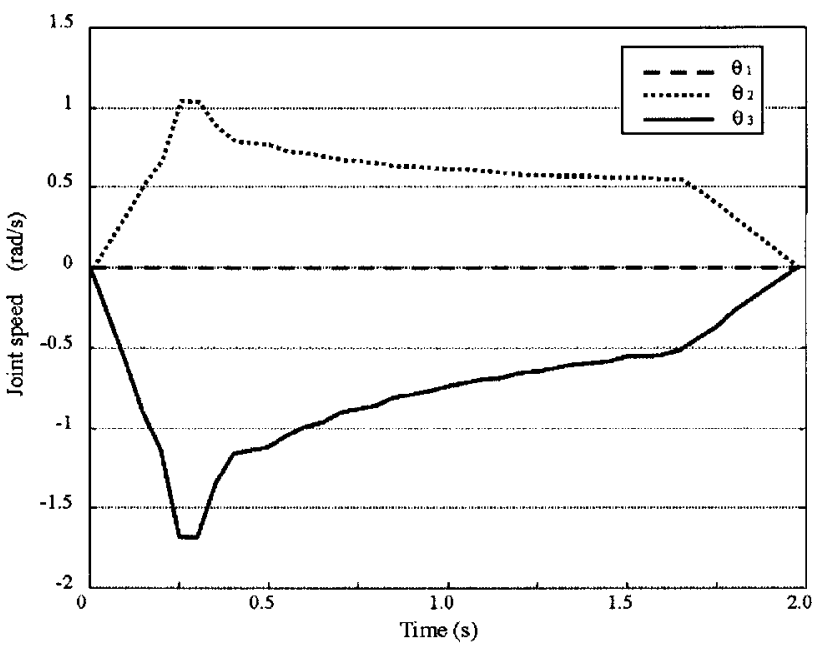

Figure 7. SILO4 leg angular joint speeds when lifting the foot from leg position $(x, y, z)=(0,350,-400)$ to $(x, y, z)=(0,350,-200)$ at $100 \mathrm{~mm} / \mathrm{s}$.

(2), and as a result it will never reach the average speed value $\nu_{m 2}$ that the second profile does.

However, there are some cases where the effect of leg dynamics critically limits foot acceleration. An earlier work ${ }^{14}$ analyzing the SILO4 leg dynamics shows that the relevant dynamics affecting the motion of such a 3 DOF articulated leg are inertial effects over the first joint of the leg due to its own acceleration, and gravitational effects over the second and third joints, respectively. These dynamic effects over the leg motion correspond to the following practical observations: When the foot trajectory has a large $z$-component increment, the plane IOF is nearly vertical and the angle $\theta$ in Figure 3 is mostly defined by the second and third joints of the leg, especially by the third joint, which reaches its maximum absolute angular speed during acceleration time in the foot-velocity profile (the maximum drive torque of the joint 3 is smaller than the second one, see Fig. 7). This means that raising the foot at high speeds amplifies the requested internal acceleration for the third axis, and thus, leg dynamics could prevent such a reference from being followed (due to its own weight, note that joints 2 and 3 are mainly affected by gravity). The same effect could affect the downward movement of the leg if it is bearing the robot's weight. Also notice that the second and third joints of the SILO4 leg have skew-axis gears (see section 2), which present nonlinearities due to frictional forces and backlash. The constant perturbation of gravity added to nonlinear frictional and backlash effects could produce oscillations at the beginning of the upward movement if the requested speed is 


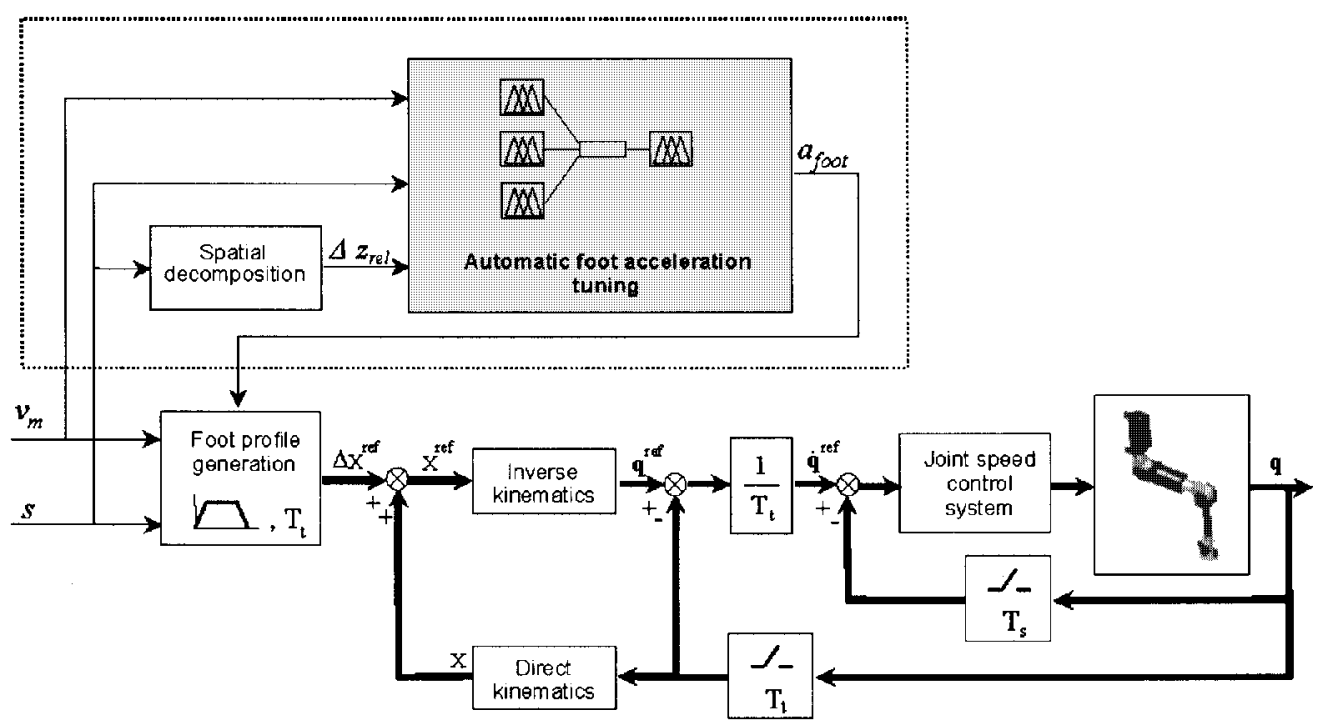

Figure 8. Block diagram of the foot trajectory control system.

high. Finding an accurate mathematical model of such dynamic effects over the leg motion is unavoidable. Fuzzy theory ${ }^{21}$ is an adequate tool for solving nonlinear system problems where a mathematical model is absent. We employed this soft computing technique to introduce the dynamics affecting the leg motion into a foot acceleration tuning algorithm that provided the best acceleration value of the foot for each trajectory.

Following this reflection we may conclude that fuzzy foot acceleration tuning as a function of the trajectory parameters and leg dynamics could be an adequate technique for improving performance in legged locomotion. Thus, the main goal of this article is to study how acceleration variations on the foot-velocity profile modify leg performance. This study will start by examining certain prior considerations, summarized as follows:

1. It is necessary to increase foot acceleration for short trajectories to obtain higher foot speeds.

2. Foot acceleration could be moderate when trajectories are large enough. A very high foot acceleration value is not needed in order to achieve a high average foot speed in the case of long trajectories. An oscillatory influence in the foot response will be avoided with moderate acceleration values.

3. Foot acceleration should be decreased for vertical movements of the foot to avoid undesired dynamic effects.
These considerations are vague rules that relate trajectory parameters with one another, although with some degree of uncertainty. In the next section, the above conditions are translated into fuzzy rules that manage the relationships between short- and long-distance trajectories, required average speed and whether or not the foot is moving upwards or downwards as input variables to infer an appropriate foot acceleration.

Figure 8 shows a block diagram of the foot trajectory control system. The foot positioning system fits into the classical scheme; further details are beyond the scope of this article. Blocks inside the dotted rectangle correspond to the foot acceleration tuning method that will be described in the next section.

\section{FUZZY SETS AND FUZZY RULES}

The problem of finding the best value of foot acceleration for a given foot trajectory is overcome by using a very simple Mamdani fuzzy inference system. ${ }^{22}$ Three input linguistic variables define foot trajectory, which are: desired average foot speed $\left(\nu_{m}\right)$, distance from initial to final position (s), and relative $z$-increment $\left(\Delta z_{\text {rel }}\right)$, which is the ratio between $z$ increment and distance traveled for a given trajectory, that is,

$$
\Delta z_{\text {rel }}=\frac{\left|z\left(t_{f}\right)-z\left(t_{i}\right)\right|}{s} \leq 1
$$


The output variable of the fuzzy inference system is the foot-acceleration, which is needed for footvelocity profile determination and thus foot trajectory generation.

Input and output fuzzy variables are represented by fuzzy sets (for example, distance is $B I G$, or foot speed is $S M A L L)$, and the degree of membership of each variable $(\mu(x)$ for the variable $x)$ to the fuzzy sets is given by membership functions. The shape of these membership functions is chosen for each linguistic variable to adjust the relationship between speed, distance and acceleration in a triangular- or trapezoidal-velocity profile, which makes acceleration inversely proportional to distance and directly proportional to the square of the speed, which is expressed thus in analytical form

$$
a_{\mathrm{foot}}=K \frac{\nu_{m}^{2}}{s}
$$

where $K$ is a constant value for each profile: $K=4$ for a triangular-velocity profile, $K>4$ for a trapezoidalvelocity profile. In this work, guidelines on fuzzy controller design have been followed. ${ }^{23}$ They state that the inference map shape matches the shape of membership functions of the input variables, provided that membership functions are normal, symmetrical and overlapped by pairs, and the membership functions defined over the output variables have the same area. Taking this into consideration, the following assumptions have been made in order to design the fuzzy system.

1. Let us assume that the relative $z$ increment in a trajectory, $\Delta z_{\text {rel }}$, is represented by two fuzzy sets $\{S M A L L, B I G\}$. Membership functions of this input variable are trapezoidal and are shown in Figure 9(a) where the abscissa is the value of the relative $z$ increment and the ordinate $\mu\left(\Delta z_{\text {rel }}\right)$ is the degree of membership. The relationship between $\Delta z_{\text {rel }}$ and $a_{\text {foot }}$ in Eq. (3) requires a negative inclination of the resulting inference map along this variable edge, which will be expressed by means of fuzzy rules.

2. Trajectory distance $(s)$ is also represented by the same fuzzy sets $\{S M A L L, B I G\}$. Two trapezoidal distance membership functions are shown in Figure 9(b), where the abscissa is the value of the trajectory distance and the ordinate $\mu(s)$ is the degree of membership. Their limit values were obtained experimentally for the SILO4 leg workspace, where the maximum linear distance for a trajectory is $700 \mathrm{~mm}$.

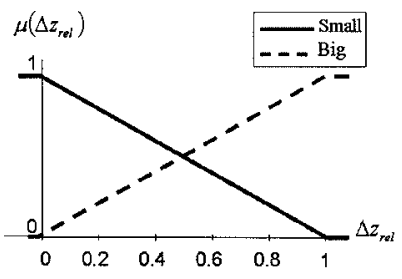

(a)

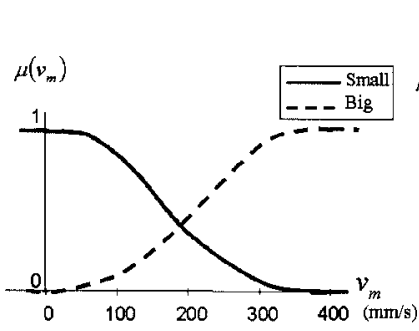

(c)

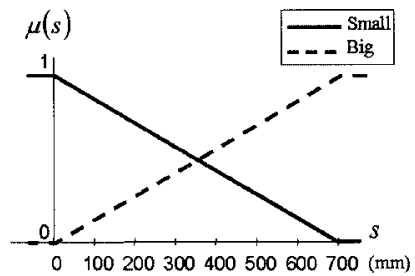

(b)

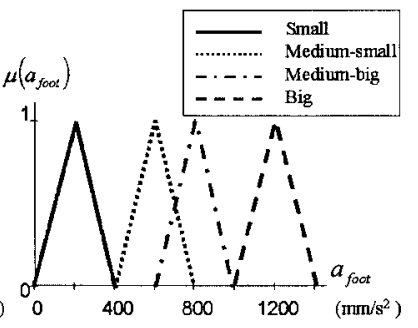

(d)
Figure 9. Membership functions of the fuzzy inference system input and output variables. (a) Relative $z$ increment; (b) Trajectory distance; (c) Average foot speed; (d) Foot acceleration.

3. Average foot speed $\left(\nu_{m}\right)$ is represented by the same fuzzy sets as the first two variables. However, membership functions are parabolic rather than trapezoidal (see Fig. 9c) just to adjust to the relationship in Eq. (3). Their limit values were found experimentally for the SILO4 leg example. The average foot speed is limited to $400 \mathrm{~mm} / \mathrm{s}$ due to maximum motor speed. A foot speed of $200 \mathrm{~mm} / \mathrm{s}$ seems to be an intermediate value appropriate for straight-line motion (see Fig. 9c).

4. The output of this fuzzy inference system is the foot acceleration $\left(a_{\text {foot }}\right)$, which is represented by four fuzzy sets $\{S M A L L$, MEDIUM-SMALL, MEDIUM-BIG, BIG\}, and membership functions are shown in Figure 9(d). These membership functions are triangular, representing $S M A L L$ foot accelerations for long trajectories without any $z$ increment, MEDIUM-SMALL acceleration values for trajectories having a big $z$ increment, MEDIUM-BIG accelerations for long trajectories with high speed, and BIG accelerations for short trajectories with high speed values. The limit values of membership functions are obtained experimentally for the SILO4 leg example.

Hence, the inference mechanism is based on the following five rules, which represent the fuzzy 
dependence of foot acceleration on foot speed, trajectory distance and relative $z$ increment:

1. If $\nu_{m}$ is $S M A L L$ and $s$ is $S M A L L$ and $\Delta z_{\text {rel }}$ is SMALL then $a_{\mathrm{foot}}$ is MEDIUM-BIG.

2. If $\nu_{m}$ is $S M A L L$ and $s$ is $B I G$ and $\Delta z_{\text {rel }}$ is SMALL then $a_{\text {foot }}$ is SMALL.

3. If $\nu_{m}$ is $B I G$ and $s$ is SMALL and $\Delta z_{\text {rel }}$ is SMALL then $a_{\text {foot }}$ is BIG.

4. If $\nu_{m}$ is BIG and $s$ is BIG and $\Delta z_{\text {rel }}$ is SMALL then $a_{\mathrm{foot}}$ is MEDIUM-BIG.

5. If $\Delta z_{\text {rel }}$ is BIG then $a_{\text {foot }}$ is MEDIUM-SMALL.

MATLAB and its Fuzzy Toolbox were used to solve the fuzzy problem, were min represents the and method and implication, max represents aggregation, and centroid is used for defuzzification. The inference map for the foot-acceleration problem, is a hypersurface which we have represented by threebidimensional inference maps for the sake of clarity. Figure 10 shows foot acceleration as a function of the distance traveled and the average foot speed for any trajectory with almost no $z$ increment in the foot trajectory, that is, when the relative $z$ increment is very close to zero. Figure 11 shows the limiting effect of any $z$ increment in foot acceleration. Figure 11(a) shows the foot-acceleration output as a function of the relative $z$ increment and distance traveled, when fixing the average foot velocity at a value of 200 $\mathrm{mm} / \mathrm{s}$, and Figure 11(b) shows acceleration versus relative $z$ increment and average foot speed, for a distance traveled of $350 \mathrm{~mm}$.

Once the foot-acceleration function has been obtained, optimization methods for real-time implementation of the fuzzy reasoning process can be used. ${ }^{24}$

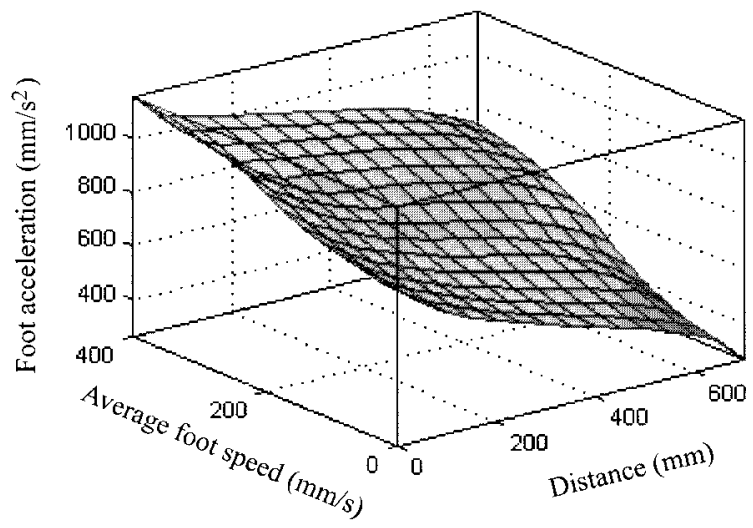

Figure 10. Foot-acceleration function of trajectory distance and average foot speed for $\Delta z_{\text {rel }}=0$.

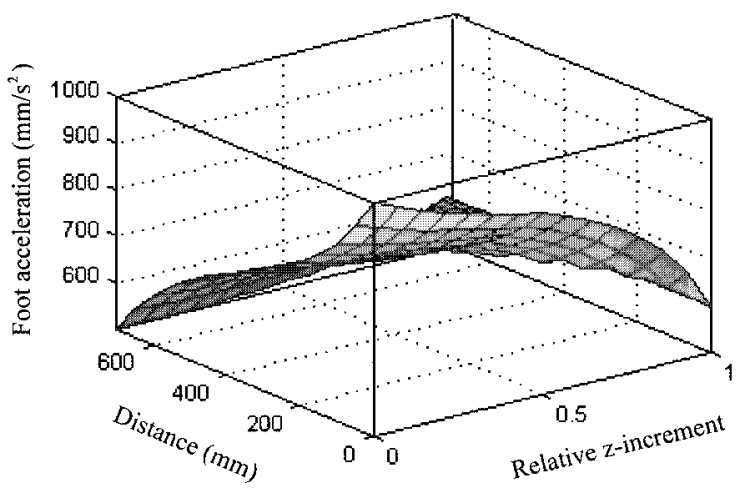

(a)

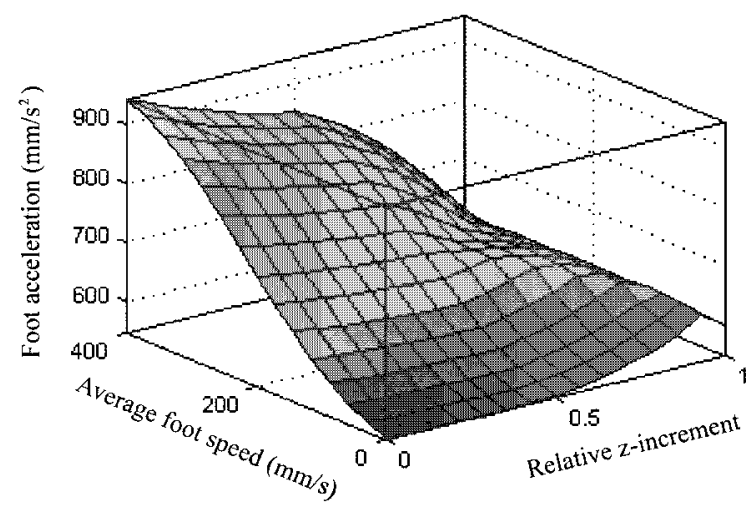

(b)

Figure 11. Relative $z$-increment effect versus (a) distance traveled and (b) foot speed, in the foot-acceleration tuning.

As it was mentioned throughout this article, the acceleration tuning method has been tailored to the SILO4 leg. In order to provide a general algorithm valid for any articulated manipulator, the following steps summarize the general procedure:

1. Workspace partioning taking limitation of drives into account. Only non-drive-limited trajectories will be subject to end-effector acceleration tuning (see section 3).

2. Experimental determination of the range of values for the trajectory parameters. That is, trajectory distance, average speed, and acceleration ranges must be identified (see section 3).

3. Trajectory parameter transfer to fuzzy linguistic variables, and finding of fuzzy sets by partitioning the universe of discourse of each variable (see section 4 ).

4. Direct application of the fuzzy inference system described in section 4 to the linguistic variables. 
The solution will be a relationship between the acceleration of the end-effector and the trajectory parameters.

\section{EXPERIMENTAL RESULTS}

Different experiments have been conducted to show the improvement on straight-line trajectory execution by foot-acceleration tuning. For this purpose the SILO4 leg has been used. The specifications of this leg are listed in section 2. The first experiment shows the effect of foot-acceleration tuning when executing straight-line trajectories of several lengths. Figure 12 illustrates this experiment, depicting the maximum achievable average foot speed for different trajectory distance. Every trajectory was parallel to the leg's $x$-axis and for $y=215 \mathrm{~mm}$ and $z=-250 \mathrm{~mm}$. Each thin curve in this graph represents maximum average foot speeds that could be reached with a foot-velocity profile of constant acceleration provided that no dynamics perturb the motion, and the thick curve represents the maximum achievable foot speed during the same trajectories using foot-acceleration tuning. If the maximum constant acceleration curves were used, the dynamics affecting the leg motion would prevent the leg from following the specified path and oscillations and non-desired effects will appear as shown in Figure 13. To ensure that leg dynamics will not perturb the motion, a conservative acceleration value should

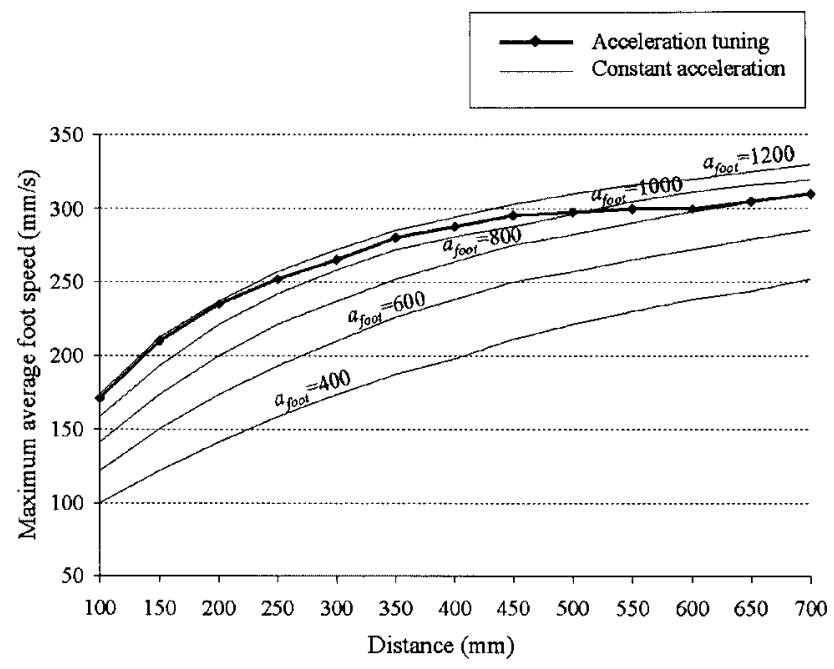

Figure 12. Curves of maximum average foot speed versus trajectory distance with constant foot-acceleration profile and with foot-acceleration tuning.

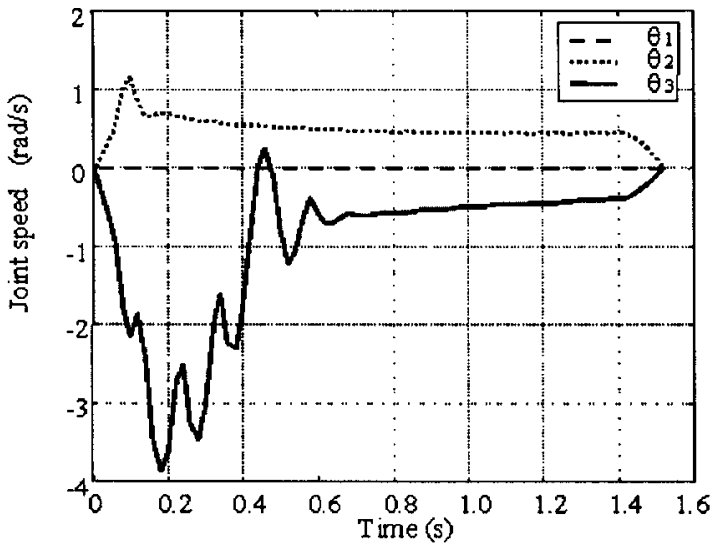

(a)

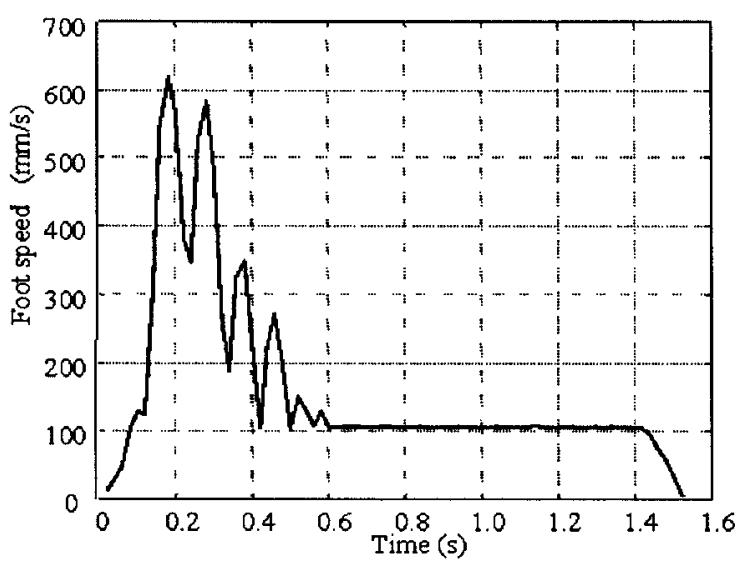

(b)

Figure 13. Leg oscillations during lifting experiments with foot-acceleration $=1000 \mathrm{~mm} / \mathrm{s}^{2}$. (a) Articular joint velocity profiles. (b) Foot-velocity profile.

be chosen (i.e. $600 \mathrm{~mm} / \mathrm{s}^{2}$ ), and low speed trajectories would be performed for distances where leg dynamics does not prevent achieving higher speeds. Figure 13 clearly shows the improvement of footacceleration tuning on this problem. One important feature of every curve $a_{\text {foot }}=$ constant is observable in this figure. The increasing rate of the curves is different before and after a foot speed of $220 \mathrm{~mm} / \mathrm{s}$. Every curve increases more slowly when crossing this line. This is clearly due to limitation of drives. $\mathrm{Up}$ to this point every foot velocity profile is triangular, and the maximum speed in the profile is double the average speed value of the trajectory. For that instant of time when the foot speed is double the average value, the joint speed will reach the maximum motor speed if

$$
\frac{2 \nu_{m}}{R\left(t_{\mathrm{a}}\right)} \cdot \frac{60}{2 \pi} \cdot N \geq R P M_{\max }
$$


where $\nu_{m}$ is the average speed value in that trajectory, $t_{\mathrm{a}}$ is the end of the acceleration time instant, $N$ is the gear ratio and $R P M_{\max }$ is the maximum motor speed in rpm. In the case of the first joint of the SILO4 leg, $R P M_{\max }=4800 \mathrm{rpm}$, and for $R=215$ $\mathrm{mm}$ and $N=246$, Eq. (4) gives a speed limit of 220 $\mathrm{mm} / \mathrm{s}$ for a triangular speed profile. To increase the foot speed above this value a trapezoidal foot speed must be selected, and that makes the change in the curve tendency.

Another important feature can be observed from Figure 12. The maximum average speed curve that is achieved using acceleration tuning (thick line in the figure) is nearly constant for BIG distance trajectories $(s>350 \mathrm{~mm})$. If this walking robot control system manages to walk always over these BIG step trajectories, the maximum robot speed will be always achieved, independently of the trajectory distance, $s$. This is not possible using a velocity profile with constant acceleration, where maximum average speed changes with trajectory distance as Figure 12 shows.

However, the maximum average speed curve using acceleration tuning is not constant at all for SMALL trajectories $(s<350 \mathrm{~mm})$. A very high acceleration would have to be used to obtain a constant curve for these short trajectories, and more powerful drives would be required for this purpose, increasing the weight of the leg, and therefore the need for more power to support the increase of weight, in a never-ending cycle.

The conclusion of this first experiment is that foot-acceleration tuning finds the acceleration values that provide higher foot speeds, avoiding the use of very high acceleration values that could impose oscillatory behavior. It also helps maintain the maximum average foot speed as a constant specification for the control system.

A second experiment was run lifting the foot at high speeds. Foot-acceleration tuning limits the acceleration value to $600 \mathrm{~mm} / \mathrm{s}^{2}$ when lifting the foot, as a result of applying the fifth fuzzy rule to $\Delta z_{\text {rel }} \approx 1$ (see Figure 11). Thus, oscillations due to leg's dynamic effects were avoided by using a moderate acceleration value. Without acceleration tuning, a foot-acceleration value higher than $600 \mathrm{~mm} / \mathrm{s}^{2}$ produces oscillations during foot lifting, due to leg weight and nonlinearities of the second and third joints of the leg. Figure 13(a) shows velocity profiles of the three joints, and Figure 13(b) shows the footvelocity profile, while the foot tried to lift its own weight at a speed of $90 \mathrm{~mm} / \mathrm{s}$ with a foot acceleration of $1000 \mathrm{~mm} / \mathrm{s}^{2}$. The angular speed of the third joint of the leg reaches its maximum achievable value (limitation of the drive) during acceleration time, which makes the leg oscillate. However, as this figure shows, there is a settling time for the leg response, when the joint speed required for the third axis decreases smoothly.

The last experiment showing the acceptability of this method studied the behavior of acceleration tuning through a variety of trajectories covering the leg workspace. Figure 14 illustrates this experiment, where trajectories of different length, $s(t)$, and different radius, $R(t)$, were executed by the leg with and without acceleration tuning at its maximum achievable foot speed (thick and thin lines, respectively). Every trajectory is confined to the plane $z=$ $-300 \mathrm{~mm}$. Behavior was the same within different z-component planes, and was only worse for those foot heights where the two last links of the leg are aligned (singularity) due to the excessive increase in internal speed during acceleration time of the second and third joints. However, this situation always appears, whether automatically tuning the acceleration or not, and it implies a reduction of foot acceleration to $600 \mathrm{~mm} / \mathrm{s}^{2}$, and thus every maximum foot speed value inside this plane of the leg workspace becomes very limited. Average foot speed values as well as distance traveled and minimum radius $R(t)$ values are listed in Table II for each trajectory of the experiment in Figure 14. The improvement offered by the acceleration tuning algorithm is relevant for short trajectories $(s<$ $350 \mathrm{~mm}$ ) and acceptable for long ones, as shown in Table II. Improvement reaches higher values for trajectories of the same length $(s(t))$ with higher $R(t)$, as stated in Eq. (1). As shown in Figure 14, leg behavior with acceleration tuning is less oscillatory for every trajectory because high average speed values can be achieved without reaching the drive limits. Also, higher average foot speeds can be achieved. Table II reveals that the acceleration tuning method increases the average foot speed by $27-100 \%$ over the maximum achievable speed when acceleration tuning is not used.

It may be concluded that the foot acceleration tuning approach for straight-line trajectory generation is a very suitable method for achieving precise, smooth, and fast foot movements, which is highly important for legged locomotion. All the experiments in this article show on-line-generated straight-line trajectories of the foot. The on-line path planning computation was realized at a sampling period of $4 \mathrm{~ms}$ on a 486 processor at $50 \mathrm{MHz}$, 


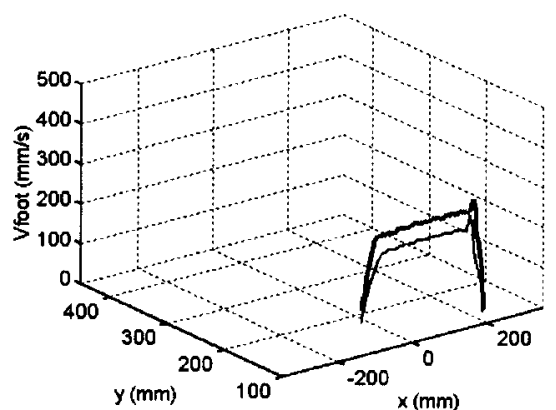

(a) $\mathrm{s}=330 \mathrm{~mm}$.

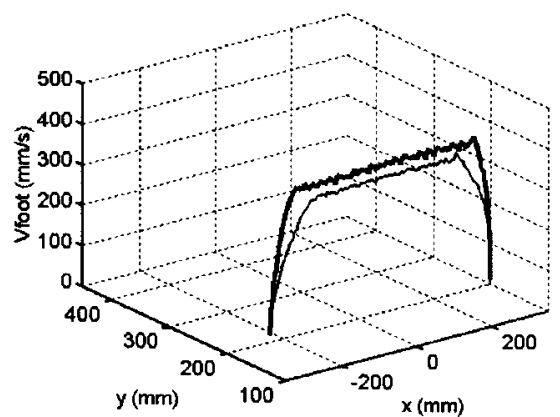

(c) $\mathrm{s}=580 \mathrm{~mm}$.

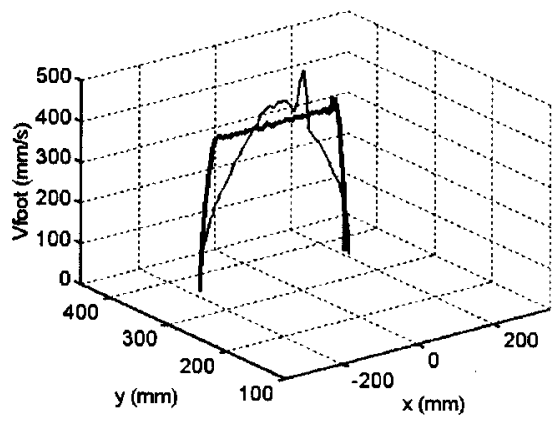

(e) $\mathrm{s}=\mathbf{4 0 0} \mathrm{mm}$.

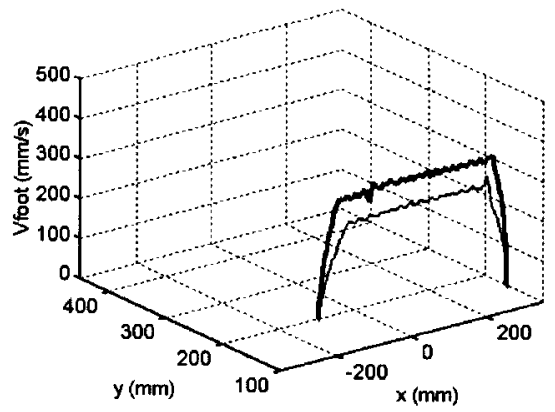

(b) $\mathbf{s}=\mathbf{5 0 0} \mathrm{mm}$

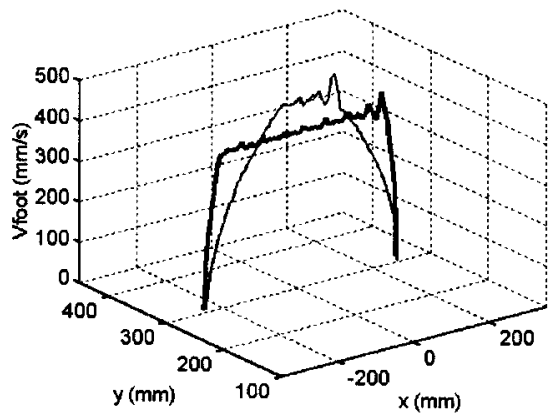

(d) $\mathrm{s}=510 \mathrm{~mm}$.

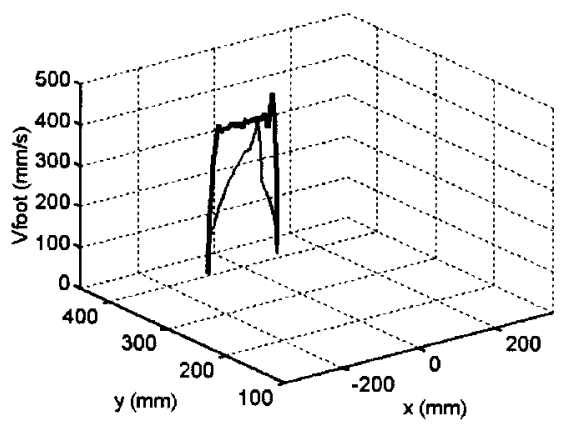

(f) $\mathrm{s}=\mathbf{2 0 0} \mathrm{mm}$.

Figure 14. Comparison between foot velocity profiles with acceleration tuning (thick line) and with constant acceleration of $600 \mathrm{~mm} / \mathrm{s}^{2}$ (thin line).

Table II. Comparison of maximum achievable average foot speeds.

\begin{tabular}{ccccc}
\hline & & \multicolumn{2}{c}{$\nu_{m}^{\max }(\mathrm{mm} / \mathrm{s})$} & \\
\cline { 3 - 4 } $\min _{t_{i} \leq t \leq t_{f}}\{R(t)\}(\mathrm{mm})$ & $s(\mathrm{~mm})$ & Acceleration tuning & Acceleration $=600 \mathrm{~mm} / \mathrm{s}^{2}$ & Improvement $(\%)$ \\
\hline 120 & 330 & 230 & 180 & 27.7 \\
150 & 500 & 290 & 220 & 31.8 \\
200 & 580 & 310 & 250 & 24.0 \\
300 & 510 & 340 & 270 & 25.9 \\
350 & 400 & 340 & 240 & 41.6 \\
400 & 200 & 320 & 160 & 100.0 \\
\hline
\end{tabular}


while the fuzzy foot acceleration tuning calculation required $0.5 \mathrm{~ms}$ before trajectory tracking. Guidelines $^{24}$ on optimal implementation of fuzzy controllers have been followed. This ensures the real-time application of the proposed acceleration tuning algorithm in the locomotion system of a walking robot. The number of fuzzy rules imposed on the foot behavior to solve the speed optimization problem can be modified in terms of dynamic complexity; however, it is independent on the number of degrees of freedom of the robot leg.

\section{CONCLUSIONS}

The work presented in this article focuses on optimizing the average leg speed of a walking robot. Several experiments have been conducted using a small walking robot to study how average foot speed can be increased. These experiments have been illustrated in detail, and the phenomenon has been thoroughly explained. Acceleration of the speed profile has been targeted as the proper magnitude to be optimized.

To avoid problems stemming from the robot's parameter uncertainties, fuzzy techniques have been used. For this purpose, fuzzy rules are defined based on experiments, and the optimal acceleration for every given trajectory is found. A simple Mamdani fuzzy inference system is used to compute the required acceleration. It is based on five rules using three linguistic variables.

Some experiments have been carried out to validate the algorithm. These experiments concluded that the foot acceleration tuning method finds the acceleration values that provide fast and smooth foot trajectories, avoiding perturbing effects due to saturation of the actuators and leg dynamics. Finally, some experiments were performed to compare the behavior of a leg executing the same trajectory both with and without the acceleration tuning method. This comparative study reveals that, depending on the distance traveled, the acceleration tuning method increases average foot speed by $27-100 \%$ over the maximum achievable speed when acceleration tuning is not used.

The real-time application of the proposed acceleration tuning algorithm in the locomotion system of a walking robot is proved. Since rules are imposed on the foot motion, the computational complexity of the algorithm is independent on the number of degrees of freedom of the robot leg. However, the number of fuzzy rules imposed over the foot behavior can increase in terms of dynamic complexity of the system.

These conclusions validate the acceleration tuning method for on-line straight trajectory generation as a good method for achieving precise, smooth, fast foot movements. This is a major improvement in walking machine locomotion now incorporated in the SILO4 walking robot.

\section{Acknowledgments}

The authors thank Fernando Matia, María Antonia Jimenez, and Jose A. Galvez for their helpful comments and suggestions.

\section{REFERENCES}

1. S. Song and K.J. Waldron, Machines that walk: The adaptive suspension vehicle, The MIT Press, Cambridge, MA, 1989.

2. P. Gonzalez de Santos and M.A. Jimenez, Generation of discontinuous gaits for quadruped walking machines, J Robotic Syst 12(9) (1995), 599-611.

3. R.B. McGhee and G.I. Iswandhi, Adaptive locomotion of a multilegged robot over rough terrain, IEEE Trans Syst, Man and Cybern 9(4) (1979), 176-182.

4. F. Delcomyn, Walking robots and the central and peripheral control of locomotion in insects, Autonomous Robots 7(3) (1999), 259-270.

5. J. Dean, T. Kindermann, J. Schmitz, M. Schumm, and $\mathrm{H}$. Cruse, Control of walking in the stick insect: From behavior and physiology to modeling, Autonomous Robots 7(3) (1999), 271-288.

6. P. Gonzalez de Santos, M.A. Armada and M.A. Jimenez, An industrial walking machine for naval construction, IEEE Int Conf Robotics Automation, Albuquerque, NM, 1997, pp. 28-36.

7. J.C. Grieco, M. Prieto, M.A. Armada, and P. Gonzalez de Santos, A six-legged climbing robot for high payloads, IEEE Int Conf Control Applications, Trieste (Italy), 1998, pp. 446-452.

8. J.E. Bares and W.L. Whittaker, Configuration of autonomous walkers for extreme terrain, The Int J Robotics Res 12(6) (1993), 535-559.

9. J. Bobrow, S. Dubowsky, and J. Gibson, Time-optimal control of robotic manipulators along specified paths. The Int J Robotics Res 4(3) (1985), 3-17.

10. K.G. Shin and N.D. Mckay. Minimum-time control of robotic manipulators with geometric constraints. IEEE Trans Autom Contr AC-30(6) (1985), 531-541.

11. F. Pfeiffer and R. Johanni. A concept for manipulator trajectory planning, IEEE J Robotics Automation RA3(2) (1987), 115-123.

12. J.-J.E. Slotine and H.S. Yang. Improving the efficiency of time-optimal path-following algorithms. IEEE Trans Robotics Automation 5(1) (1989), 118-124. 
13. Z. Shiller. On singular points and arcs in path constrained time optimal motions. ASME, Dyn Syst Contr Division DSC-42 42 (1992), 141-147.

14. H. Yang and J.-J. Slotine, Fast algorithms for nearminimum-time control of robot manipulators. The Int J Robotics Res 13(6) (1994), 521-532.

15. E. García, J.A. Gálvez, and P. González de Santos, A mathematical model for real-time control of the SILO4 leg, 3rd Int Conf CLAWAR, Madrid, Spain, 2000, pp. 447-460.

16. L. Pan and P.Y. Woo, PD manipulator controller with fuzzy adaptive gravity compensation, J Robotic Syst 17(2) (2000), 93-106.

17. L.C. Lin and G.Y. Lee, Hierarchical fuzzy control for $\mathrm{C}$-axis of CNC turning centers using genetic algorithms, J Intell Robotic Syst 25(3) (1999), 255-275.

18. J.A. Galvez, P. Gonzalez de Santos, and M. Armada,
A force controlled robot for agile walking on rough terrain, IFAC Workshop on Intelligent Components for Vehicles, Seville, Spain, 1998, pp. 247-252.

19. M. Vukobratovic, Introduction to robotics, SpringerVerlag, Berlin, 1989, pp. 81-82.

20. J.J. Craig, Introduction to robotics, 2 nd ed., AddisonWesley, Reading, MA, 1989.

21. H.J. Zimmermann, Fuzzy set theory and its applications, 2nd ed., Kluwer Academic Publishers, Dordrecht, 1991.

22. E.H. Mamdani, Fuzzy reasoning and its applications, Academic Press, New York, 1981.

23. F. Matia, A. Jimenez, R. Galán, and R. Sanz, Fuzzy controllers: Lifting the linear-nonlinear frontier, Fuzzy Sets Syst 52(2) (1992), 113-128.

24. F. Matia and A. Jimenez, On optimal implementation of fuzzy controllers, Int J Intell Contr Syst 1(3) (1996), 407-415. 